\title{
Quantum stabilization of classically unstable plateau structures
}

\author{
Tommaso Coletta, ${ }^{1}$ M. E. Zhitomirsky, ${ }^{2}$ and Frédéric Mila ${ }^{1}$ \\ ${ }^{1}$ Institute of Theoretical Physics, Ecole Polytechnique Fédérale de Lausanne (EPFL), CH-1015 Lausanne, Switzerland \\ ${ }^{2}$ Service de Physique Statistique, Magnétisme et Supraconductivité, UMR-E9001 CEA-INAC/UJF, 17 rue des Martyrs, \\ F-38054 Grenoble, France
}

(Received 12 December 2012; published 19 February 2013)

\begin{abstract}
Motivated by an intriguing report, in some frustrated quantum antiferromagnets, of magnetization plateaus whose simple collinear structure is not stabilized by an external magnetic field in the classical limit, we develop a semiclassical method to estimate the zero-point energy of collinear configurations even when they do not correspond to a local minimum of the classical energy. For the spin- $1 / 2$ frustrated square-lattice antiferromagnet, this approach leads to the stabilization of a large $1 / 2$ plateau with "up-up-up-down" structure for $J_{2} / J_{1}>1 / 2$, in agreement with exact diagonalization results, while for the spin-1/2 anisotropic triangular antiferromagnet, it predicts that the $1 / 3$ plateau with "up-up-down" structure is stable far from the isotropic point, in agreement with the properties of $\mathrm{Cs}_{2} \mathrm{CuBr}_{4}$.
\end{abstract}

DOI: 10.1103/PhysRevB.87.060407

PACS number(s): 75.10.Jm, 75.30.Ds, 75.50.Ee

Introduction. Frustration is responsible for the emergence of several remarkable properties in quantum magnets, ranging from rather exotic types of order such as quadrupolar or nematic order to resonating valence bond or algebraic spin liquids. ${ }^{1}$ In the presence of an external field, frustration is also known to be at the origin of several types of accidents in the magnetization curve, including kinks, jumps, and plateaus. Of all these remarkable features, magnetization plateaus at a rational value of the magnetization are probably the best documented ones experimentally, and their theory is likewise quite advanced. Following the terminology of Hida and Affleck, ${ }^{2}$ two kinds of plateaus have been identified: ${ }^{3}$ "classical" plateaus, ${ }^{4-6}$ whose structure has a simple classical analog with spins up or down along the external field, and "quantum" plateaus, ${ }^{7-11}$ which have no classical analog and correspond to a Wigner crystal of triplets in a sea of singlets. In the case of quantum plateaus, the mechanism is clear: Frustration reduces the kinetic energy of triplets, resulting in a crystallization at commensurate densities. The main open problem is to be predictive for high commensurability plateaus since it requires a precise knowledge of the long-range part of the triplet-triplet interaction.

By contrast, and somehow surprisingly, the theory of classical plateaus is not yet complete. The paradigmatic example of a classical plateau is the $1 / 3$ magnetization plateau of the Heisenberg antiferromagnet on a triangular lattice, studied by Chubukov and Golosov ${ }^{5}$ in the context of a $1 / S$ expansion. In this system the three-sublattice up-up-down (uud) structure appears classically at $H=H_{\text {sat }} / 3$, and since, according to order by disorder, collinear configurations tend to have a softer spectrum, hence a smaller zero-point energy, ${ }^{12,13}$ quantum fluctuations stabilize this $u и d$ state in a finite field range around $H_{\text {sat }} / 3$, leading to the $1 / 3$ plateau. This prediction has been confirmed by exact diagonalization of finite clusters for $S=1 / 2$ and $1,{ }^{14}$ and the theory of Chubukov and Golosov can be extended to all cases where a collinear state is classically stabilized for a certain field.

There are cases, however, where a classical plateau has been suggested to exist although the collinear structure stabilized for quantum spins is not the ground state for classical spins, the classical ground state in the appropriate field range being in general a noncoplanar structure. This is, for instance, the case of the spin-1/2 $J_{1}-J_{2}$ model on the square lattice, for which exact diagonalizations have revealed the presence of a foursublattice up-up-up-down (иииd) $1 / 2$ plateau in a parameter range where the classical ground state has a canted stripe structure (see below). Another example is the $1 / 3$ plateau of the Heisenberg model on the anisotropic triangular lattice, a model relevant to the compound $\mathrm{Cs}_{2} \mathrm{CuBr}_{4}$. To develop a general theory of classical plateaus in that situation remains the main open issue in the field.

The goal of this Rapid Communication is to develop such a theory. For that purpose, we start with a general Heisenberg model in an external field defined by the spin Hamiltonian

$$
\mathcal{H}=\sum_{\langle i, j\rangle} J_{i j} \mathbf{S}_{i} \cdot \mathbf{S}_{j}-H \sum_{i} S_{i}^{z},
$$

and show how to estimate the zero-point energy of collinear states even if they do not minimize the classical energy. More precisely, we derive an upper bound of this energy to order $1 / S$. If the energy of a collinear state estimated in this way is lower than that of the classical ground state (including the zero-point energy), then the collinear state must be the ground state. Accordingly, the quantum antiferromagnet exhibits a magnetization plateau in a certain field range, which may generally exceed our conservative theoretical estimate based on the upper energy bound. We apply this approach to the $J_{1}-J_{2}$ model on a square lattice and to the Heisenberg antiferomagnet on the anisotropic triangular lattice, with results in remarkable agreement with existing numerical data for $S=1 / 2$.

General formalism. In the semiclassical approach, deviations from the classical configuration are expressed as Holstein Primakoff bosons. ${ }^{15}$ At the harmonic level, the bosonic Hamiltonian can be split into three contributions $\mathcal{H}=\mathcal{H}^{(0)}+\mathcal{H}^{(1)}+\mathcal{H}^{(2)}$, where $\mathcal{H}^{(0)}$ is the classical energy of the system, while $\mathcal{H}^{(1)}$ and $\mathcal{H}^{(2)}$ respectively contain only terms which are linear and quadratic in boson operators. Now, $\mathcal{H}^{(1)}=0$ if the configuration minimizes the classical energy, but more generally this holds true if it is a saddle point of the energy, a condition fulfilled by plateau structures since they are collinear. In all these cases, the fluctuations are 
described by a purely quadratic Hamiltonian. However, since plateau structures are classical minima of the energy only at specific values of couplings and magnetic field (if any), the resulting quadratic Hamiltonian is positive definite only at these specific points. Away from such points the correction to the classical energy cannot be computed at the harmonic level, and if the plateau state is to become the true quantum ground state, higher-order terms in the spin-wave expansion must be included to produce an excitation spectrum with positive frequencies, as done in Ref. 5 and in more recent studies. ${ }^{16,17}$ This is a rather involved calculation, and it would be useful to have a simpler approach to determine if there is a plateau, and to estimate its width to the lowest order in $1 / S$. Besides, the calculation of the excitation spectrum for the plateau region allows to identify only second-order transitions, ${ }^{5}$ while in many experimental and model examples the transitions at the plateau boundaries are of the first order and, therefore, require a full energetic comparison.

To obtain a well-defined spectrum around a state which is not a classical ground state we propose to add a staggered-field term,

$$
\hat{V}=\delta \sum_{i}\left(S-S_{i}^{z_{i}}\right)
$$

to the harmonic Hamiltonian. A similar approach has been introduced in a different context in Refs. 18 and 19. On each lattice site $i$, the staggered field $\delta>0$ is oriented in the direction $\hat{\mathbf{z}}_{i}$ of the corresponding classical spin. The extra term (2) does not change the classical energy and simply amounts to a shift of the chemical potential of the Holstein-Primakoff bosons $\hat{V}=\delta \sum_{i} a_{i}^{\dagger} a_{i}$, which yields a positive contribution to the spin-wave Hamiltonian. The magnitude of $\delta$ is adjusted to the minimal value that ensures that the harmonic Hamiltonian is positive definite, so that the resulting spectrum obtained with the help of the Bogolyubov transformation has real and positive frequencies. Since the expectation value of $\hat{V}$ is strictly positive, the energy calculated adding $\hat{V}$ provides an upper bound for the energy of the plateau state.

$J_{1}-J_{2}$ model. For the frustrated square-lattice antiferromagnet the exchange interaction constants are $J_{i j}=J_{1}$ and $J_{2}$ for nearest and second-nearest neighbors, respectively. In zero field the classical ground state of the model is a helix with the ordering wave vector given by the minimum of the Fourier transform of the coupling interaction $J_{\mathbf{q}}=4 J_{1} \gamma_{\mathbf{q}}+4 J_{2} \eta_{\mathbf{q}}$, with $\gamma_{\mathbf{q}}=\left(\cos q_{x}+\cos q_{y}\right) / 2$ and $\eta_{\mathbf{q}}=\cos q_{x} \cos q_{y}$. For $J_{2} / J_{1}<1 / 2$, the minimum corresponds to $\mathbf{q}_{N}=(\pi, \pi)$, i.e., to Néel order. In the opposite case $J_{2} / J_{1}>1 / 2$, the orderby-disorder mechanism selects collinear striped structures with ordering wave vectors $\mathbf{q}_{S}=(\pi, 0)$ or $(0, \pi) .{ }^{13}$ The point $J_{2} / J_{1}=1 / 2$ is highly degenerate since $J_{\mathbf{q}}$ is minimal along the lines $q_{x}=\pi$ and $q_{y}=\pi$. In the presence of a magnetic field both the Néel and the stripe structure are canted with a uniform spin component in the field direction. The canting angle $\theta$ measured with respect to the $z$ axis is given by $\cos \theta_{N}=H / 8 J_{1} S$ and $\cos \theta_{S}=H /\left(4 J_{1}+8 J_{2}\right) S$ for the two states.

The analysis of classical spin configurations in a magnetic field suggests the appearance of a 1/2-magnetization plateau with a four-sublattice uu $\mathrm{d}$ structure for the strongly frustrated point $J_{2} / J_{1}=1 / 2 .^{6}$ The conclusion has been supported by exact diagonalizations of finite clusters, though numerically the plateau extends well into the classically unstable region $J_{2} / J_{1}>1 / 2$ with the largest width at $J_{2} / J_{1} \approx 0.6$. Moreover, the linear spin-wave calculation for $J_{2} / J_{1}=1 / 2$ and $H=$ $4 J_{1} S$ shows that for this ratio of coupling constants the canted Néel state wins over the collinear plateau state, ${ }^{20}$ leaving an apparent problem with reconciling numerical and analytical results.

We now investigate the appearance of the $1 / 2$ magnetization plateau for the $J_{1}-J_{2}$ model using the variational harmonic theory outlined above to estimate the energy of the uuud structure, as well as that of the canted Néel state for $J_{2} / J_{1}>1 / 2$ and of the stripe structure for $J_{2} / J_{1}<1 / 2$, where these configurations are saddle points of the classical energy. The general structure of the quadratic bosonic Hamiltonian is given by

$$
\mathcal{H}=N E_{\mathrm{cl}}+\frac{1}{2} \sum_{\mathbf{k}}\left[\hat{\mathbf{a}}_{\mathbf{k}}^{\dagger} M_{\mathbf{k}} \hat{\mathbf{a}}_{\mathbf{k}}-\Delta_{\mathbf{k}}\right],
$$

where $E_{\mathrm{cl}}$ is the classical energy per site of the state around which fluctuations are considered. For the canted Néel and the canted stripe states $\hat{\mathbf{a}}_{\mathbf{k}}^{\dagger}=\left(a_{\mathbf{k}}^{\dagger}, a_{-\mathbf{k}}\right)$ and $M_{\mathbf{k}}$ is the $2 \times 2$ matrix,

$$
M_{\mathbf{k}}(\mathbf{q})=\left(\begin{array}{ll}
A_{\mathbf{k}}(\mathbf{q}) & B_{\mathbf{k}}(\mathbf{q}) \\
B_{\mathbf{k}}(\mathbf{q}) & A_{\mathbf{k}}(\mathbf{q})
\end{array}\right),
$$

with

$$
\begin{aligned}
& A_{\mathbf{k}}\left(\mathbf{q}_{N}\right)=4 J_{1} S\left(1+\gamma_{\mathbf{k}} \cos ^{2} \theta_{N}\right)-4 J_{2} S\left(1-\eta_{\mathbf{k}}\right), \\
& B_{\mathbf{k}}\left(\mathbf{q}_{N}\right)=-4 J_{1} S \gamma_{\mathbf{k}} \sin ^{2} \theta_{N}
\end{aligned}
$$

for the Néel state and

$A_{\mathbf{k}}\left(\mathbf{q}_{S}\right)=4 J_{2} S\left(1+\eta_{\mathbf{k}} \cos ^{2} \theta_{S}\right)+2 J_{1} S\left(\cos k_{y}+\cos ^{2} \theta_{S} \cos k_{x}\right)$,

$B_{\mathbf{k}}\left(\mathbf{q}_{S}\right)=-2 S \sin ^{2} \theta_{S}\left(J_{1} \cos k_{x}+2 J_{2} \eta_{\mathbf{k}}\right)$

for the stripe state, with $\mathbf{q}_{S}=(\pi, 0)$. The additional constants $\Delta_{\mathbf{k}}^{N}$ and $\Delta_{\mathbf{k}}^{S}$ in Eq. (3) are given by $A_{\mathbf{k}}\left(\mathbf{q}_{N}\right)$ and $A_{\mathbf{k}}\left(\mathbf{q}_{S}\right)$.

The uuud state has a four-site unit cell, and $\hat{\mathbf{a}}_{\mathbf{k}}^{\dagger}$ denotes $\left(a_{1, \mathbf{k}}^{\dagger}, \ldots, a_{4, \mathbf{k}}^{\dagger}, a_{1,-\mathbf{k}}, \ldots, a_{4,-\mathbf{k}}\right)$, with $M_{\mathbf{k}}$ being the $8 \times 8$ matrix obtained from (4) by substituting

$A_{\mathbf{k}}=\left(\begin{array}{cccc}\bar{A}_{\mathbf{k}} & \bar{E}_{\mathbf{k}} & 0 & \bar{G}_{\mathbf{k}}^{\star} \\ \bar{E}_{\mathbf{k}}^{\star} & \bar{B}_{\mathbf{k}} & 0 & \bar{F}_{\mathbf{k}} \\ 0 & 0 & \bar{C}_{\mathbf{k}} & 0 \\ \bar{G}_{\mathbf{k}} & \bar{F}_{\mathbf{k}}^{\star} & 0 & \bar{A}_{\mathbf{k}}\end{array}\right), B_{\mathbf{k}}=\left(\begin{array}{cccc}0 & 0 & -\bar{F}_{\mathbf{k}} & 0 \\ 0 & 0 & \bar{H}_{\mathbf{k}}^{\star} & 0 \\ -\bar{F}_{\mathbf{k}}^{\star} & \bar{H}_{\mathbf{k}} & 0 & -\bar{E}_{\mathbf{k}} \\ 0 & 0 & -\bar{E}_{\mathbf{k}}^{\star} & 0\end{array}\right)$,

with coefficients

$$
\begin{aligned}
& \bar{A}_{\mathbf{k}}=-4 J_{2} S+H, \quad \bar{B}_{\mathbf{k}}=-4\left(J_{1}-J_{2}\right) S+H, \\
& \bar{C}_{\mathbf{k}}=4\left(J_{1}+J_{2}\right) S-H, \quad \bar{E}_{\mathbf{k}}=J_{1} S \tau_{k_{y}}, \\
& \bar{F}_{\mathbf{k}}=J_{1} S \tau_{k_{x}}, \quad \bar{G}_{\mathbf{k}}=J_{2} S \tau_{-k_{x}} \tau_{-k_{y}}, \quad \bar{H}_{\mathbf{k}}=-J_{2} S \tau_{-k_{x}} \tau_{k_{y}},
\end{aligned}
$$

where $\tau_{k}=\left(1+e^{-i 2 k}\right)$. The constant $\Delta_{\mathbf{k}}^{u u u d}$ is given by $2 \bar{A}_{\mathbf{k}}+$ $\bar{B}_{\mathbf{k}}+\bar{C}_{\mathbf{k}}$. When the matrix $M_{\mathbf{k}}$ is not positive definite we add to the Hamiltonian the term $\hat{V}$ defined in Eq. (2), whose effect is to increase $\Delta_{\mathbf{k}}$ and all diagonal elements of $M_{\mathbf{k}}$ by $\delta / 2$, with a field $\delta$ adjusted to the minimal value that makes $M_{\mathbf{k}}$ positive definite over the entire Brillouin zone. 


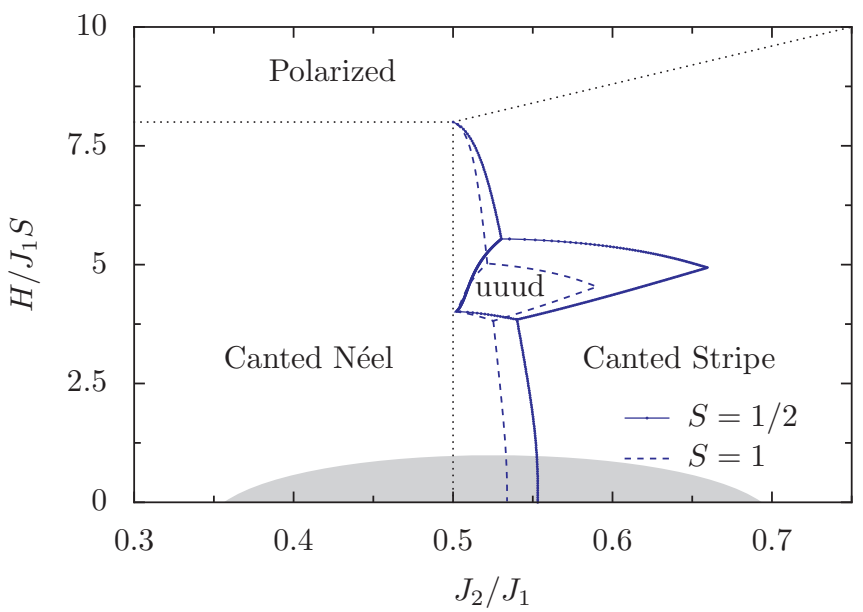

FIG. 1. (Color online) Semiclassical phase diagram for the spin $1 / 2 J_{1}-J_{2}$ Heisenberg model in magnetic field. The $u u u d$ structure is stabilized by fluctuations over a wide parameter range. Dashed lines correspond to the phase diagram for $S=1$ and dotted lines to the classical phase boundaries. The shaded area represents schematically the gapped singlet phase for the spin- $1 / 2$ model.

The phase diagram obtained by comparing the ground-state energies for three relevant spin structures is presented in Fig. 1 for $S=1 / 2$ and 1 . The 1/2-magnetization plateau is stabilized by quantum fluctuations over a wide range of parameters deep into the classically forbidden region $J_{2} / J_{1}>1 / 2$, though it remains energetically unfavorable at $J_{2} / J_{1}=1 / 2$. The width and position of the plateau are in good agreement with the exact diagonalization results of finite clusters with up to $N=$ 36 sites. $^{6}$ Figure 2 shows the magnetization curves for several ratios $J_{2} / J_{1}$. The magnetization curve for $J_{2} / J_{1}=0.6$ with a large magnetization jump below the plateau and a much smaller anomaly above the plateau is in good correspondence with the numerical data for the same coupling ratio. ${ }^{6}$ For $J_{2} / J_{1}$ close to $1 / 2$, there is, in addition, a competition between the canted Néel and the canted stripe states. The Néel state has a softer spectrum than the stripe state and is stabilized beyond its classical boundary. This leads to an additional transition from the canted Néel state into the canted stripe structure which shows up as a small jump either above $\left(J_{2} / J_{1}=0.525\right)$ or below $\left(J_{2} / J_{1}=0.55\right)$ the $1 / 2$ plateau.

It should be pointed out that states other than those considered may be stabilized. One possible candidate, at the

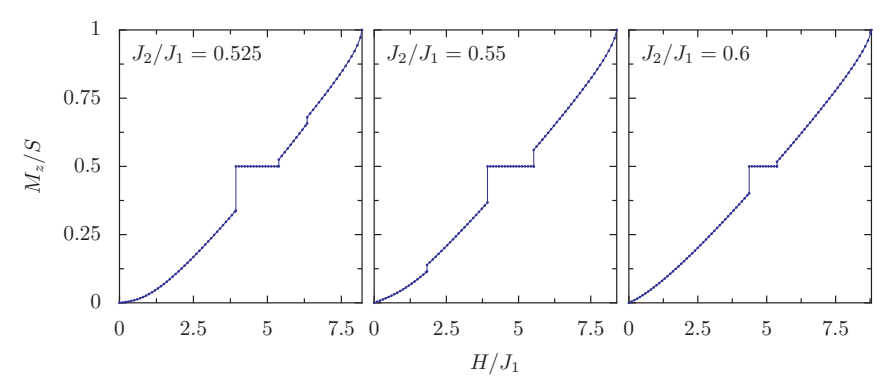

FIG. 2. (Color online) Magnetization curves of the spin $1 / 2 J_{1}-J_{2}$ model for different ratios $J_{2} / J_{1}$ obtained in the variational spin-wave approach.

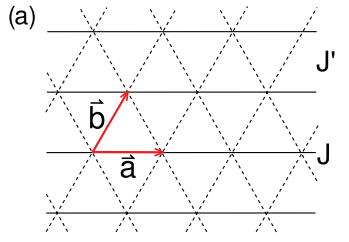

(b) 3 sublattice planar states

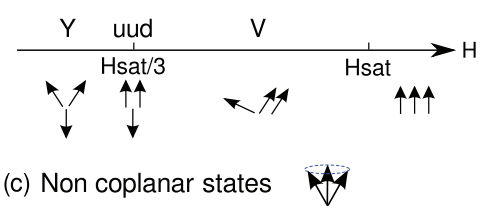

FIG. 3. (Color online) (a) Anisotropic triangular lattice. (b) Three-sublattice planar structure as a function of the magnetic field. (c) Example of noncoplanar canted helix.

upper edge of the plateau, is the coplanar four sublattice state having three classical spins parallel and the remaining spin pointing in a different direction. This state can be naturally connected to the uuud state and is the analog of the state stabilized above the plateau in the isotropic triangular lattice. However, such a structure could not be investigated in our linear spin-wave approach since it is not a saddle point of the classical energy.

Anisotropic triangular lattice. We now consider a second example of a classically unstable magnetization plateau, the nearest-neighbor Heisenberg antiferromagnet on an orthorhombically distorted (anisotropic) triangular lattice. In this model spins are coupled by $J_{i j}=J$ along horizontal chains and by $J_{i j}=J^{\prime}$ on zigzag interchain bonds [see Fig. 3(a)]. The spin-1/2 model is relevant for $\mathrm{Cs}_{2} \mathrm{CuBr}_{4},{ }^{21-23}$ which has a $1 / 3$ magnetization plateau, although, with $J^{\prime} \sim 0.75 J$, it is quite far from the isotropic limit. The robustness of the $u u d$ plateau in the $J-J^{\prime}$ model has been studied numerically ${ }^{24,25}$ and analytically. ${ }^{16}$ Nevertheless, the extent of the plateau state around $H / H_{\text {sat }} \sim 1 / 3$ and $J^{\prime} / J \sim 1$ and the nature of the states adjacent to the plateau region are still open questions. Furthermore, the stability method employed by Alicea et al. ${ }^{16}$ allows to identify only second-order transitions out of the plateau state, while experiments typically find first-order transitions, ${ }^{23}$ a fact naturally explained in our theoretical approach (see below).

The Fourier transform of the coupling interaction in the triangular lattice [see Fig. 3(a) $]$ is given by $J_{\mathbf{q}}=2[J \cos \mathbf{q a}+$ $\left.J^{\prime} \cos \mathbf{q b}+J^{\prime} \cos \mathbf{q}(\mathbf{a}-\mathbf{b})\right]$. In zero field the classical ground state is a helical spin structure whose ordering wave vector Q minimizes $J_{\mathbf{q}}$. In the isotropic case $J=J^{\prime}$ this yields the well-known $120^{\circ}$ spin structure. In the presence of a magnetic field the classical energy is minimized for canted helices or umbrella configurations [see Fig. 3(c)], which have helical order in the $x y$ plane and a uniform spin component in the field direction. The canting angle of the helical structure measured with respect to the $z$ axis is given by $\cos \theta_{H}=H /\left(J_{0}-J_{\mathbf{Q}}\right) S$. For the isotropic point $J^{\prime} / J=1$ the canted helical state is degenerate with the coplanar Y- and V-type structures [see Fig. 3(b)]. Existing linear spin-wave calculations indicate that the coplanar structures are selected over the noncoplanar one in the isotropic lattice and that the uud structure, classically stable at the field $H_{\text {sat }} / 3$, is stabilized by fluctuations over a finite field range. ${ }^{5}$

In the following we address the problem of the plateau stability for the anisotropic triangular lattice model by comparing ground-state energies for the canted helical state, the uud structure and the two three-sublattice planar states as a function of $J^{\prime} / J$ and magnetic field. Away from $J=J^{\prime}$, 


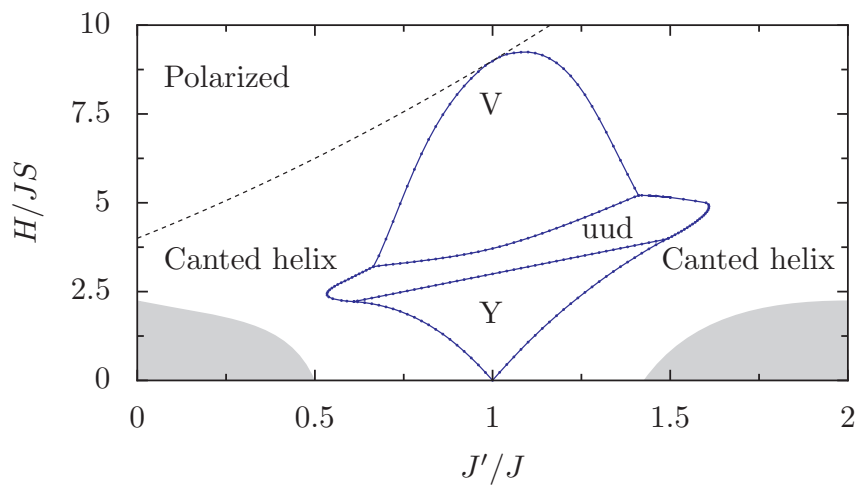

FIG. 4. (Color online) Phase diagram of the spin-1/2 anisotropic triangular lattice in magnetic field. $\mathrm{Y}$ and $\mathrm{V}$ regions denote threesublattice planar states. The dashed line is the classical saturation field. The gray shading denotes regions where phases other than the canted helical states may be expected.

the three-sublattice planar structures turn out to be, as the $u u d$ state away from $H_{\text {sat }} / 3$ and $J=J^{\prime}$, saddle points but not local minima of the classical energy, and to compute their zero-point energies, we again use the variational spin-wave approach suggested above. ${ }^{26}$

The resulting phase diagram is shown in Fig. 4. The uud plateau state is stabilized well beyond the isotropic limit and extends over the range $0.5 \lesssim J^{\prime} / J \lesssim 1.5$. Coplanar states are stabilized above and below the magnetization plateau with the exception of the plateau edges, where we find direct first-order transitions from the $u u d$ state into the canted helical structure. From the energetic comparison it appears that the uud state does not extend into the Y-state region, so that the corresponding portion of the lower boundary of the plateau is perfectly linear (see Fig. 4). This is almost certainly an artefact of the method, which only gives an upper bound to the energy of the plateau, and the extent of the plateau is probably significantly larger. In fact, at $J=J^{\prime}$, we obtain a plateau width which is only half that predicted in Refs. 5 and 14 . We also note that the only coplanar states considered in our calculation are Y- and V-type structures, while for a substantial mismatch between $J$ and $J^{\prime}$, incommensurate coplanar structures may be also stabilized by quantum fluctuations. The variational spin-wave approach is not well suited for treating them and we only remark that they may appear on the phase diagram at the expense of the canted helical structure. Finally, the gray shading in Fig. 4 indicates regions where new quantum phases are expected. In fact, in zero field, theoretical and numerical approaches point to collinear spin correlations for weakly coupled chains, ${ }^{27-29}$ while in the limit of strong interchain couplings the antiferromagnetic Néel state should be stable down to $J^{\prime} / J \approx 1.5$. $^{29}$

As compared to those of Alicea et al., who also predicted an extended plateau region for small distortions $\left[\left(1-J^{\prime} / J\right)^{2} \lesssim\right.$ $0.3]$ for the $S=1 / 2$ case $^{16}$ our results bring in a number of additional insights. In the first place, the symmetry between $J^{\prime} / J<1$ and $J^{\prime} / J>1$ is lost. Second, a transition out of the plateau into the canted helical states is clearly present. Finally, for $J^{\prime} / J=0.75$ relevant for $\mathrm{Cs}_{2} \mathrm{CuBr}_{4},{ }^{21-23}$ we find a magnetization plateau width $\Delta H / H_{\text {sat }} \approx 0.106$, significantly larger than the experimental value $\Delta H / H_{\text {sat }} \approx 0.052$ in $\mathrm{Cs}_{2} \mathrm{CuBr}_{4}$. Since by construction our approach tends to underestimate the plateau width, the difference must be due to additional effects not included in the anisotropic model, for instance, the competition between quantum effects and Dzyaloshinskii-Moriya interactions. ${ }^{30}$

Conclusion. We have developed a general method to investigate the stabilization of classical magnetization plateaus in cases where the corresponding configuration is not a minimum of the classical energy. This method is extremely simple since it only relies on the diagonalization of quadratic bosonic Hamiltonians and does not require to go beyond linear spin-wave theory, yet it appears to give remarkably accurate results, even for spin $1 / 2$. This has been demonstrated in two cases of current interest, the $J_{1}-J_{2}$ Heisenberg model on the square lattice and the Heisenberg model on the anisotropic triangular lattice, for which it predicts that plateaus at magnetization $1 / 2$ and $1 / 3$, respectively, are stabilized over a wide range of parameters.

Acknowledgments. We acknowledge useful discussions with Andrey Chubukov, Sergey Korshunov, and Karlo Penc. This work has been supported by the Swiss National Fund and by MaNEP.

\footnotetext{
${ }^{1}$ Introduction to Frustrated Magnetism, edited by C. Lacroix, P. Mendels, and F. Mila (Springer, Berlin, 2011).

${ }^{2}$ K. Hida and I. Affleck, J. Phys. Soc. Jpn. 74, 1849 (2005).

${ }^{3}$ For a recent review, see M. Takigawa and F. Mila, in Magnetization Plateaus in Introduction to Frustrated Magnetism, edited by C. Lacroix, P. Mendels, and F. Mila (Springer, Berlin, 2011), p. 241.

${ }^{4}$ H. Kawamura, J. Phys. Soc. Jpn. 53, 2452 (1984).

${ }^{5}$ A. V. Chubukov and D. A. Golosov, J. Phys.: Condens. Matter 3, 69 (1991).

${ }^{6}$ M. E. Zhitomirsky, A. Honecker, and O. A. Petrenko, Phys. Rev. Lett. 85, 3269 (2000).

${ }^{7}$ K. Totsuka, Phys. Rev. B 57, 3454 (1998).

${ }^{8}$ F. Mila, Eur. Phys. J. B 6, 201 (1998).

${ }^{9}$ S. Miyahara and K. Ueda, Phys. Rev. Lett. 82, 3701 (1999).
}

${ }^{10}$ J. Dorier, K. P. Schmidt, and F. Mila, Phys. Rev. Lett. 101, 250402 (2008).

${ }^{11}$ A. Abendschein and S. Capponi, Phys. Rev. Lett. 101, 227201 (2008).

${ }^{12}$ E. F. Shender, JETP 56, 178 (1982).

${ }^{13}$ C. L. Henley, Phys. Rev. Lett. 62, 2056 (1989).

${ }^{14}$ A. Honecker, J. Schulenburg, and J. Richter, J. Phys.: Condens. Matter 16, S749 (2004).

${ }^{15}$ T. Holstein and H. Primakoff, Phys. Rev. 58, 1098 (1940).

${ }^{16}$ J. Alicea, A. V. Chubukov, and O. A. Starykh, Phys. Rev. Lett. 102, 137201 (2009)

${ }^{17}$ J. Takano, H. Tsunetsugu, and M. E. Zhitomirsky, J. Phys.: Conf. Ser. 320, 012011 (2011).

${ }^{18}$ T. Coletta, S. E. Korshunov, and F. Mila (unpublished).

${ }^{19}$ S. Wenzel, T. Coletta, S. E. Korshunov, and F. Mila, Phys. Rev. Lett. 109, 187202 (2012). 
${ }^{20}$ G. Jackeli and M. E. Zhitomirsky, Phys. Rev. Lett. 93, 017201 (2004).

${ }^{21}$ T. Ono, H. Tanaka, O. Kolomiyets, H. Mitamura, T. Goto, K. Nakajima, A. Oosawa, Y. Koike, K. Kakurai, J. Klenke et al., J. Phys.: Condens. Matter 16, S773 (2004).

${ }^{22}$ H. Tsujii, C. R. Rotundu, T. Ono, H. Tanaka, B. Andraka, K. Ingersent, and Y. Takano, Phys. Rev. B 76, 060406 (2007).

${ }^{23}$ N. A. Fortune, S. T. Hannahs, Y. Yoshida, T. E. Sherline, T. Ono, H. Tanaka, and Y. Takano, Phys. Rev. Lett. 102, 257201 (2009).

${ }^{24}$ S. Miyahara, K. Ogino, and N. Furukawa, Physica B 378-380, 587 (2006).
${ }^{25}$ R. Chen, H. Ju, H.-C. Jiang, O. A. Starykh, and L. Balents, arXiv:1211.1676.

${ }^{26}$ See Supplemental Material at http://link.aps.org/supplemental/ 10.1103/PhysRevB.87.060407 for details concerning the spin-wave calculation in the case of the anisotropic triangular lattice AFM.

${ }^{27}$ O. A. Starykh and L. Balents, Phys. Rev. Lett. 98, 077205 (2007).

${ }^{28}$ A. Weichselbaum and S. R. White, Phys. Rev. B 84, 245130 (2011).

${ }^{29}$ J. Reuther and R. Thomale, Phys. Rev. B 83, 024402 (2011).

${ }^{30}$ C. Griset, S. Head, J. Alicea, and O. A. Starykh, Phys. Rev. B 84, 245108 (2011) 